Pensamiento Crítico N. ${ }^{\circ}$ 9, pp. 27-44

\title{
Globalización económica y pobreza
}

Econ. Abel Andrés Zavala

Lic. Ciro E. Calero Castillo

\section{RESUMEN}

El artículo aborda la relación entre globalización y pobreza por ingresos. El análisis toma como punto de partida la información existente sobre la evaluación de la pobreza desde 1820 en adelante. Luego, se estudia los dos canales mediante los cuales la globalización afecta a la pobreza: El Directo y el Indirecto. Este último ocurre a través del crecimiento económico. No es simple separar los efectos de la mayor integración económica sobre el mayor o menor crecimiento, pues este último no depende solo de la integración. Existe una multitud de factores que lo condicionan, muchos de los cuales no se ubican directamente en la esfera económica. En lo que sí existe un mayor consenso es en la necesidad de crecer para reducir pobreza. Esto no significa que el consenso se ubique en torno a cómo crecer.

Palabras clave: Globalización como sinónimo de internalización, globalización como sinónimo de la liberación económica, globalización como sinónimo de occidentalización o modernización, globalización económica y pobreza, globalización económica al crecimiento. 


\title{
Abel Andrés Zavala / Ciro E. Calero Castillo
}

\begin{abstract}
The article is about the relation between globalization and poverty by incomes. The analysis takes, as a starting point, the existing information about the evaluation of poverty since 1820 and on. Then both channels have to be studied through wich the globalization affects poverty: The Direct and the Indirect, the second one happens because of the increasing of the economy. It is not that simple to separate the effects of the greatest economical integration over greatest or smallest increasing, since this last one does not depend on integration only. There is a dozen of factors that determine it, a lot of wich are not placed directly in the economical sphere. Where there is a great consensus is in the necessity of increasing to reduce poverty. This does not mean that the consensus is located around "How to grow".
\end{abstract}

Keywords: Globalization.

\section{INTRODUCCIÓN}

La globalización económica -es decir, la mayor integración económica mundiales un medio para elevar el Bienestar de los habitantes del planeta y no un fin en si misma. La pobreza es una dimensión del bienestar, estrechamente relacionada con la distribución de ingresos.

El objetivo fundamental de este artículo es relacionar la globalización económica con la pobreza monetaria, es decir, la pobreza por ingreso.

Por otra parte, los críticos de la globalización apuntan a la extrema rapidez de la apertura en todos los campos, en el sentido de que la profundidad y la velocidad de la apertura generan efectos perniciosos sobre la pobreza, que son difíciles de enfrentar por los gobiernos, pues el mismo proceso globalizador reduce los grados de maniobra que tienen para enfrentar la pobreza. Por su lado, los pro globalizadotes sostienen lo contrario, es decir, que la integración es imprescindible para reducir la pobreza. En esta parte existe consenso en que se requiere de políticas complementarias para magnificar el impacto positivo sobre la disminución de la pobreza. En cualquier caso, lo que no puede perderse de vista es el contexto sociopolítico dentro del que operan las fuerzas globalizadotas, pues aunque existiera un acuerdo respecto de cual posición es la correcta, el siguiente paso, relacionado con la implementación de las políticas enfrenta dificultades mayores aún. 


\section{Globalización económica y pobreza}

En lo que ambas posiciones coinciden es en la urgente necesidad de enfrentar la reducción de la pobreza. El consenso es universal. En setiembre del año 2000, 189 estados miembros de las Naciones Unidas firmaron un compromiso mundial para el desarrollo, cuya expresión política quedó reflejada en la declaración del milenio. Su primer objetivo es la reducción del porcentaje de pobreza extrema a la mitad entre 1990 y el año 2015.

\section{RELACIÓN ENTRE GLOBALIZACIÓN ECONÓMICA Y POBREZA}

La globalización afecta a la pobreza a través de dos canales:

- Canal Directo. En lo comercial, la apertura altera los precios relativos que enfrentan los pobres, como consumidores y como productores. Si la liberación lleva a una reducción de los precios de los bienes comprados por los pobres, entonces la globalización reduce la pobreza. En paralela, si la misma eleva los precios de los bienes producidos por los pobres, entonces el efecto también tendrá la misma dirección. En cuanto a la apertura financiera, los efectos sobre la reducción de la pobreza dependerán del tipo de capital que ingrese al país; por ejemplo, si se trata de inversión extranjera directa, en función del sector al cual se oriente, aumentaría la demanda por cierto tipo de trabajadores que ostentan los niveles de calificación requeridos por los inversionistas.

- Canal Indirecto. Sostiene que la mayor integración genera mayores tasas de crecimiento económico y que a través de este se impacta en la reducción de la pobreza; el nexo entre estos últimos es, básicamente, el empleo; además, como el crecimiento económico aumenta, la recaudación tributaria eleva la capacidad de gasto del gobierno de turno; si este es destinado a sectores sociales, entonces contribuirá con la disminución de la pobreza. Aquí el supuesto implícito es que el gobierno invierta adecuadamente los recursos obtenidos.

\section{INTERPRETANDO LA GLOBALIZACIÓN}

Un hecho real es que la popularidad del concepto es relativamente reciente, pues viene un par de décadas atrás. Coincide con el final de la Guerra Fría, con la caída del muro de Berlín, con el colapso del socialismo de Estado o de planificación central, es decir, con la desaparición de la Unión Soviética, y el descomunal avance de las tecnologías 


\section{Abel Andrés Zavala / Ciro E. Calero Castillo}

de la información, por lo tanto, la difusión del vocablo adquirió dimensiones globales o planetarias al mismo tiempo que ocurrían hechos políticos de relevancia para la historia de la humanidad. De ahí que, a pesar de que el término es perfectamente usado en su dimensión económica, tenga una connotación política. Esta lleva a pensar en una asociación entre el capitalismo sistema, neoliberalismo estrategia y globalización proceso; además, el término NEOLIBERALISMO se hizo popular desde inicios de la década de 1980, al mismo tiempo que el vocablo GLOBALIZACIÓN.

Held y Mc Grew (2002) denominan a los primeros Globalistas; y a los segundos, Escépticos. En un libro previo, los mismos autores utilizan la acepción Hiperglobalistas, en lugar de globalistas (Held, Mc Grew, Goloblat y Perraton, 1999); no obstante, al tratarse de posiciones extremas, no solo caben interpretaciones intermedias, sino que existen variantes dentro de cada una de ellas. Por ejemplo, no todo los globalistas se adhieren al neo liberalismo, algunos se relacionan con la social democracia. Lo mismo ocurre, aunque con menor tendencia.

\section{GLOBALIZACIÓN COMO SINÓNIMO DE INTERNACIONALIZACIÓN}

En esta parte, el término se refiere al aumento de la interdependencia e interconexión entre personas de distintos países. En la medida en que esta tendencia se ha acentuado en las últimas décadas, es comprensible la idea de utilizarlas como sinónimo, algunos prefieren usar el término Globalización, en lugar de Internacionalización para enfatizar la gradual erosión de los Estados-Naciones y del alcance y efectividad de las medidas diseñadas e implementadas por los mismos.

Así, las tendencias actuales representan un aumento de la internacionalización y de triadización, en torno de tres grandes bloques: Los Estados Unidos, Europa occidental y Japón. Algunos van mas allá y sugieren que la globalización no es más que un discurso para justificar el proyecto global neoliberal y la consolidación del capitalismo norteamericano en las demás regiones del mundo.

\section{GLOBALIZACIÓN COMO SINÓNIMO DE LIBERALIZACIÓN ECONÓMICA}

La globalización se refiere a un proceso de apertura de las economías, a través de la remoción de los obstáculos al comercio y a los flujos de capitales como barreras 


\section{Globalización económica y pobreza}

comerciales, restricciones cambiarias, controles de capitales etc. Puesto de otro modo alude a la integración económica global a partir del libre mercado. Esta definición es utilizada por los neoliberales, así como por sus críticos.

El uso de esta definición implica que el estudio de la globalización lleva al debate sobre los pro y los contra de las políticas económicas liberales, que caracterizan al mundo desde hace un par de décadas. Por un lado los pro globalizadores sostienen que la aplicación de los principios liberales proporcionará prosperidad, paz y democracia para todos. Por otro, los antiglobalizadores se oponen a las políticas liberales, pues sostienen que el libre mercado genera pobreza, desigualdad, conflictos sociales, destrucción cultural, daños ecológicos y déficit democrático. Desde esa óptica, el liberalismo económico sería una fuerza impulsora de los periodos de globalización, si esto es así los movimientos antiglobalizadores en su gran mayoría, estarían en contra el liberalismo como forma de organización de la economía mundial.

Como consecuencia, no debe sorprender que los movimientos antiglobalización rehagan al liberalismo y no a la globalización en sí misma. Los debates relacionados con los efectos del libre mercado tienen una larga historia y en muchos de ellos no se ha lisado la expresión "globalización"; la tendencia a equiparar "globalización" con "liberalismo" es reciente.

\section{GLOBALIZACIÓN COMO SINÓNIMO DE OCCIDENTALIZACIÓN O MO- DERNIZACIÓN}

De acuerdo con la Real Academia Española, por "occidente" se entiende a un conjunto formado por los Estados Unidos y diversos países que comparten básicamente un mismo sistema social, económico y cultural.

De allí que, en este caso, la globalización se entienda como un tipo particular de universalización; aquella en la que las estructuras sociales de la modernidad se expanden por todo el mundo desminuyendo las culturas preexistentes, así como la autodeterminación local. Equivale entonces a colonización y americanización. Puesto hegemónico que lleva a una situación de subordinación. Puesto de otro modo, se trataría de un proyecto de expansión del liberalismo. 


\section{Abel Andrés Zavala / Ciro E. Calero Castillo}

De modernidad se refiere a los modos de vida u organización social que surgieron en Europa desde alrededor del siglo XVIII en adelante y cuya influencia, posteriormente, los han convertido en más o menos mundiales; vistas la cosas así, la modernidad es intrínsecamente globalizadora. Esto no implica que sea inclusiva, pues amplios segmentos de la población mundial mantienen instituciones que pertenecen al mundo tradicional y que se encuentran fuera del circuito capitalista.

No obstante, una cosa es seguir que la globalización y la occidentalización están interconectadas, y, otra distinta, tratarlas como equivalentes. Además, la globalización puede tomar direcciones no occidentales, por lo que podía hablarse de una globalización islámica, budista, etc. De allí que la equivalencia entre ambos conceptos sea discutible. Más aún, la difusión intercontinental del budismo, cristianismo e islamismo ocurrió varios siglos antes de la formación de los Estados Unidos de América, que en realidad es un producto de la globalización capitalista de los siglos XVII y XVIII. Lo que ocurre es que la actual ola de la globalización, está fuertemente influida, al menos para América latina, por los Estados Unidos.

\section{LA REALIDAD EMPÍRICA: ANTES Y AHORA}

Los niveles globales de pobreza por ingresos, medidos a través del índice de recuento, muestran el porcentaje de pobres como proporción de la población mundial. El Cuadro N . $^{\circ}$ presenta la evolución de la pobreza en diversos años del periodo comprendido entre 1820 - 1992. Las definiciones de "pobreza" y "pobreza extrema" corresponden a las líneas de pobreza de US\$ 2 y US\$1, expresadas en dólares constantes de 1985, cabe hacer una precisión: las líneas de pobreza fueron estimadas por el Banco Mundial (Bourguignon y Morrison, 2002).

- En 1820 , el porcentaje de pobres fue de $94.4 \%$ de la población mundial, mientras que la proporción de pobres ascendió a 83,9\%. Si combinamos esta información con la reportada por los mismos autores con relación a la desigualdad global de ingresos, así como, por ejemplo: que se indique un coeficiente de Gini de 0.50 para

el mismo año, la conclusión es clara: el mundo no solo era desigual a inicios del siglo $\mathrm{XIX}$, sino que los niveles de pobreza eran altísimos. 
Globalización económica y pobreza

Cuadro $\mathrm{N}^{\circ}{ }^{1}$. Pobreza y pobreza extrema (en porcentajes y número de pobres)

\begin{tabular}{|l|c|c|c|c|c|c|c|c|}
\hline & $\mathbf{1 8 2 0}$ & $\mathbf{1 8 5 0}$ & $\mathbf{1 9 1 0}$ & $\mathbf{1 9 2 9}$ & $\mathbf{1 9 5 0}$ & $\mathbf{1 9 7 0}$ & $\mathbf{1 9 8 0}$ & $\mathbf{1 9 9 2}$ \\
\hline Porcentaje de pobres & 94,4 & 92,5 & 82,4 & 75,9 & 71,9 & 60,1 & 55,0 & 51,3 \\
\hline $\begin{array}{l}\text { Número de pobres } \\
\text { (mills) }\end{array}$ & 99,8 & 1,110 & 1,416 & 1,550 & 1,805 & 2,200 & 2,426 & 2,800 \\
\hline $\begin{array}{l}\text { Porcentaje de pobres } \\
\text { extremos }\end{array}$ & 83,9 & 81,50 & 65,6 & 56,3 & 54,8 & 35,6 & 31,5 & 23,7 \\
\hline $\begin{array}{l}\text { Número de pobres } \\
\text { extremos (mills) }\end{array}$ & 88,7 & 979 & 1,128 & 1,150 & 1,376 & 1,304 & 1,390 & 1,294 \\
\hline $\begin{array}{l}\text { Población mundial } \\
\text { (mills) }\end{array}$ & 1,057 & 1,201 & 1,719 & 2,042 & 2,511 & 3,665 & 4,414 & 5,460 \\
\hline
\end{tabular}

Fuente: Adaptado de Bourguignon y Morrison (2002:731).

- En segundo lugar, una mirada a la tendencia de la pobreza en el siglo XIX muestra una reducción del porcentaje de pobres, de $94,4 \%$ en 1820 a 82,4\%, en 1910; sin embargo, el número de pobres en el mismo periodo aumentó de 998 a 1415 millones (en el caso de los pobres extremo, el aumento fue de 887 a 1128 millones en el mismo periodo).

- En tercer lugar, de acuerdo al Cuadro N. ${ }^{\circ} 1$, entre 1820 y 1980, el porcentaje de pobres disminuyó de $94.4 \%$ a $55.0 \%$, pero el número de pobres extremos, la tendencia es similar: el porcentaje se redujo de $83.9 \%$ a $13.5 \%$, pero el número de pobres extremos aumentó de 887 a 1390 millones de habitantes.

- En cuarto lugar, para el periodo comprendido entre 1981 y 2001, conocido como "la segunda ola globalizadora", el porcentaje de pobres en los países en desarrollo se redujo de $66.7 \%$ a $52.9 \%$ (ver Cuadro ${ }^{\circ}{ }^{\circ} 2$ ), pero el número de pobres aumentó de 2.450 a 2.735 millones (ver Cuadro N. ${ }^{\circ}$ 4). Esto ocurrió a pesar de la reducción en el número de pobres en China, que pasó de 876 a 593 millones. En el caso de los pobres extremos, la reducción en porcentaje fue de $40.4 \%$ a $21.1 \%$ (ver Cuadro N. ${ }^{\circ} 3$ ). En este caso, el número de pobres mostró la misma tendencia, pues disminuyó de 1.483 a 1.089 millones (ver Cuadro N. $^{\circ}$ 5). Sin embargo, una observación por regiones 


\section{Abel Andrés Zavala / Ciro E. Calero Castillo}

permite notar que la reducción se debió casi exclusivamente a China, que redujo el número de pobres extremos e 634 a 212 millones.

- En quinto lugar, la evolución hacia la reducción de la pobreza no ha sido uniforme entre las regiones. En 1981, en Asia Oriental, el 57.7\% de la población era pobre extremo por encima del porcentaje de pobres extremos en el África. Veinte años después, el porcentaje en Asia Oriental se había reducido a 14.9\% solo en China, la disminución fue de $63.8 \%$ en 1981 a 16.6\% en el año 2001 (ver Cuadro 3) como consecuencia, el número de pobres extremo se redujo en Asia Oriental, pero aumentó en Asia Central, Europa del Este, América Latina y África (ver cuadro 5).

Cuadro N. ${ }^{\circ}$ 2. Países en desarrollo. Porcentaje pobres extremos: gasto menor de US $\$$ 1,08 diarios (1993).

\begin{tabular}{|l|c|c|c|c|c|c|}
\hline \multicolumn{6}{|c|}{ Porcentaje pobres extremos: gasto menor de US\$ 1,08 diarios (1993) } \\
\hline & $\mathbf{1 9 8 1}$ & $\mathbf{1 9 8 4}$ & $\mathbf{1 9 9 0}$ & $\mathbf{1 9 9 6}$ & $\mathbf{1 9 9 9}$ & $\mathbf{2 0 0 1}$ \\
\hline Asia Oriental & 84,8 & 76,6 & 69,9 & 53,3 & 50,3 & 47,4 \\
\hline China & 88,1 & 78,5 & 72,6 & 53,4 & 50,1 & 40,7 \\
\hline Asia Oriental sin China & 76,2 & 72,0 & 63,2 & 53,2 & 50,8 & 49,2 \\
\hline Europa Oriental y Asia Central & 4,7 & 4,1 & 4,9 & 20,7 & 23,8 & 19,7 \\
\hline América Latina y El Caribe & 26,9 & 30,4 & 28,4 & 24,1 & 25,1 & 24,5 \\
\hline Medio Oriente y África del Norte & 28,9 & 25,2 & 21,4 & 22,3 & 24,3 & 23,2 \\
\hline Asia del Sur & 89,1 & 87,2 & 85,5 & 81,7 & 78,1 & 77,2 \\
\hline India & 89,6 & 88,2 & 86,1 & 85,2 & 80,6 & 79,9 \\
\hline Asia del Sur sin la India & 87,3 & 84,0 & 83,5 & 71,3 & 70,6 & 69,0 \\
\hline África Subsahariana & 73,3 & 76,1 & 75,0 & 75,1 & 76,0 & 76,6 \\
\hline Total & 66,7 & 63,7 & 60,8 & 55,5 & 54,4 & 52,9 \\
\hline Total sin China & 58,8 & 58,4 & 56,6 & 56,3 & 55,8 & 54,9 \\
\hline
\end{tabular}

Fuente: Chen y Ravallido (2004). 


\section{Globalización económica y pobreza}

Cuadro N. ${ }^{\circ}$ 3. Países en desarrollo. Porcentaje de pobres extremos: gasto menor de US\$1,08 diarios (1993).

\begin{tabular}{|l|c|c|c|c|c|c|}
\hline & $\mathbf{1 9 8 1}$ & $\mathbf{1 9 8 4}$ & $\mathbf{1 9 9 0}$ & $\mathbf{1 9 9 6}$ & $\mathbf{1 9 9 9}$ & $\mathbf{2 0 0 1}$ \\
\hline Asia Oriental & 57,7 & 38,9 & 29,6 & 16,6 & 15,7 & 14,9 \\
\hline China & 63,8 & 41,0 & 33,0 & 17,4 & 17,8 & 16,6 \\
\hline Asia Oriental sin China & 42,0 & 33,5 & 21,1 & 14,7 & 11,0 & 10,8 \\
\hline Europa Oriental y Asia Central & 0,7 & 0,5 & 0,5 & 4,3 & 6,3 & 3,6 \\
\hline America Latina y El Caribe & 0,7 & 11,8 & 11,3 & 10,7 & 10,5 & 9,5 \\
\hline Medio Oriente y África del Norte & 5,1 & 3,8 & 2,3 & 2,0 & 2,6 & 2,4 \\
\hline Asia del Sur & 51,5 & 46,8 & 41,3 & 36,6 & 32,2 & 31,3 \\
\hline India & 54,4 & 49,8 & 42,1 & 42,2 & 35,3 & 34,7 \\
\hline Asia del Sur sin la India & 42,2 & 37,0 & 38,7 & 19,7 & 22,9 & 21,0 \\
\hline África Subsahariana & 41,6 & 46,3 & 44,6 & 45,6 & 45,7 & 46,4 \\
\hline Total & 40,4 & 32,8 & 27,9 & 22,8 & 21,8 & 21,1 \\
\hline Total sin China & 31,7 & 29,8 & 26,1 & 24,6 & 23,1 & 22,5 \\
\hline
\end{tabular}

Fuente: Chen y Ravallido (2004).

Cuadro N. ${ }^{\circ}$ 4. Países en desarrollo. Número de pobres: gasto menor de US $\$ 1,08$ diarios.

\begin{tabular}{|l|c|c|c|c|c|c|}
\hline & $\mathbf{1 9 8 1}$ & $\mathbf{1 9 8 4}$ & $\mathbf{1 9 9 0}$ & $\mathbf{1 9 9 6}$ & $\mathbf{1 9 9 9}$ & $\mathbf{2 0 0 1}$ \\
\hline Asia Oriental & 796 & 562 & 472 & 287 & 282 & 271 \\
\hline China & 634 & 425 & 375 & 212 & 223 & 212 \\
\hline Europa Oriental y Asia Oriental & 3 & 2 & 2 & 20 & 30 & 17 \\
\hline América Latina y El Caribe & 36 & 46 & 49 & 52 & 54 & 50 \\
\hline Medio Oriente y África del Norte & 9 & 8 & 6 & 6 & 8 & 7 \\
\hline Asia del Sur & 475 & 460 & 462 & 461 & 429 & 431 \\
\hline India & 382 & 374 & 357 & 400 & 352 & 359 \\
\hline África Subsahariana & 164 & 198 & 227 & 271 & 294 & 313 \\
\hline Total & 1,483 & 1,276 & 1,218 & 1,097 & 1,097 & 1,089 \\
\hline
\end{tabular}

Fuente: Chen y Ravallion (2004). 


\section{Abel Andrés Zavala / Ciro E. Calero Castillo}

- La notable redución de la pobreza extrema en China no ha sido lineal entre 1981 y 1987, el número de habitantes chinos que salió de la pobreza extrema fue de 326 millones; mientras que entre 1987 y el año 2001, la reducción de la pobreza extrema alcanzó a 96 millones. Esta observación cobra relevancia, pues la mayor disminución de la pobreza en China ocurrió antes de la implementación de las reformas comerciales (Ravallión y Chen, 2004). Si excluimos a China, el número de pobres extremos aumentó de 849 a 877 millones de habitantes en el mundo en desarrollo entre 1981 y el año 2001. Más aún, como puede verse en el Cuadro 5, la reducción en la pobreza extrema entre 1981 y 1987 fue de 311 millones de habitantes, mientras que entre 1993 y el año 2001, fue de 118 millones.

Cuadro N. ${ }^{\circ}$ 5: Países en desarrollo. Número de pobres: gasto menor de UD $\$ 2,15$ diarios.

\begin{tabular}{|l|r|r|r|r|r|r|}
\hline & $\mathbf{1 9 8 1}$ & $\mathbf{1 9 8 4}$ & $\mathbf{1 9 9 0}$ & $\mathbf{1 9 9 6}$ & $\mathbf{1 9 9 9}$ & $\mathbf{2 0 0 1}$ \\
\hline Asia Oriental & 1,170 & 1,109 & 1,116 & 922 & 900 & 864 \\
\hline China & 876 & 814 & 825 & 650 & 628 & 593 \\
\hline Europa Oriental y Asia Central & 20 & 18 & 23 & 98 & 113 & 93 \\
\hline America Latina y El Caribe & 99 & 119 & 125 & 117 & 127 & 128 \\
\hline Medio Oriente y África del Norte & 25 & 50 & 51 & 61 & 70 & 70 \\
\hline Asia Del Sur & 821 & 859 & 958 & 1,029 & 1,039 & 1,064 \\
\hline India & 630 & 661 & 732 & 806 & 804 & 826 \\
\hline Africa Subsahariana & 280 & 326 & 382 & 447 & 489 & 516 \\
\hline Total & 2,450 & 2,841 & 2,655 & 2,674 & 2,738 & 2,735 \\
\hline
\end{tabular}

Fuente: Chen y Ravallido (2004).

En síntesis, hacia el año 2001, el número de pobres en los países en desarrollo era de 2735 millones (54,9\%), mientras que el número de pobres extremos llegaba a 1089 millones $(22,5 \%)$.

El Cuadro 5 sintetiza la evolución del número de pobres y pobres extremos en los países en desarrollo durante la segunda ola globalizadora (1981-2001). Como puede observarse, si se excluye a china, tanto el número de pobres como el número de pobres 


\section{Globalización económica y pobreza}

extremos han aumentado. En consecuencia, no se puede aludir a una tendencia global en la reducción de la pobreza. Si además se excluye a la india, los resultados mostrarían un deterioro mayor. China y la india representa el $38 \%$ de la pobreza mundial, y ambos han crecido rápidamente (India, en la última década; y China, desde 1980). Dada la importancia poblacional de ambos países, cuando se realizan cálculos globales, no es difícil que su actuación repercuta en los resultados.

Cuadro N. ${ }^{\circ}$ 6. Número de pobres en los países en desarrollo (1981-2001) en millones de habitantes

\begin{tabular}{|l|r|r|}
\hline & $\mathbf{1 9 8 1}$ & $\mathbf{2 0 0 1}$ \\
\hline Pobres & 2.450 & 2.735 \\
\hline Pobres (sin China) & 1.574 & 2.142 \\
\hline Pobres extremos & 1.483 & 1.089 \\
\hline Pobres extremos (sin China) & 849 & 877 \\
\hline
\end{tabular}

Fuente: Elaboración propia.

En la medida que la globalización económica afecte a la pobreza de manera indirecta; para fines metodológicos, es necesario analizar dos "cajas negras": La primera está referida a la vinculación entre la integración económica y el crecimiento. La segunda alude a la relación entre el crecimiento y la revolución de la pobreza.

\section{GLOBALIZACIÓN ECONÓMICA INFLUYE AL CRECIMIENTO}

La globalización económica influye sobre el crecimiento a través de distintos canales, pues la primera diversas dimensiones, como la comercial, la financiera y la laboral, entre otras.

\section{INTEGRACIÓN COMERCIAL Y CRECIMIENTO ECONÓMICO}

La integración comercial alude a la reducción de los aranceles y otras trabas no-arancelarias; este proceso, como tendencia, es una característica de la 


\section{Abel Andrés Zavala / Ciro E. Calero Castillo}

economía mundial, acentuado durante las dos últimas décadas del siglo anterior. Por su parte, el crecimiento económico se define como el aumento en el PBI y/o en el PBI por habitante. La relación entre ambos conceptos ha sido objeto de amplio debate. Por un lado, una corriente de opinión sostiene que la mayor integración comercial conduce a un mayor crecimiento, lo que se discute es la relación entre el ritmo de la apertura comercial y su impacto sobre el crecimiento. Los países que liberalizan más rápido, ¿crecen más? Si la respuesta es afirmativa, entonces la mayor globalización comercial, esto es la mayor integración, es el camino a seguir.

Durante la década de 1990, la convicción de que la apertura comercial es positiva para el crecimiento económico fue sostenida en una serie de estudios de corte transversal, entre los que destacan los de David Dollar (1922), Rodríguez y Rodrik (2000) criticaron los hallazgos anteriores sobre la base de los débiles fundamentos empíricos, así como de las medidas de apertura utilizadas y las deficiencias econométricas.

Los autores relacionan el crecimiento del PBI por habitante con los cambios en el ratio comercio/PBI, que es usado como una medida de apertura comercial para ello; tomando como punto de partida el año 1980, dividen a 73 países en desarrollo en dos grupos: los más globalizados, definidos como aquellos que han mostrado aumentos dramáticos en el ratio comercial/PBI, así como declinaciones en los aranceles; y aquellos menos globalizados son aquellos que se ubican en el tercio superior de la muestra, en términos de aumento en el ratio comercial/PBI entre 1975-1979 y 1995-1997 a partir de la reducción en los aranceles entre 1985-1989 y 1995-1997 en otros términos, identifican con aquellos que se han mantenido "más cerrados".

Por otro lado, el primer grupo, el ratio comercial/PBI, aumentó de $16 \%$ a $33 \%$ del PBI entre 1980 y 2000, y los aranceles se redujeron, en promedio, de $57 \%$ a $35 \%$. En paralelo, las tazas de crecimiento por habitantes, en promedio, aumentaron de $3.5 \%$ en la década de 1980 a $5.0 \%$ en la década siguiente. El otro grupo creció $0.8 \%$ y $1.4 \%$, respectivamente, mientras que el ratio comercial/PBI disminuyó de $60 \%$ a 49\%; de ahí, concluyen que los países que más se abrieron al comercio internacional mostraron mayores tasas de crecimiento. 


\section{Globalización económica y pobreza}

Las conclusiones de Dollar y Kraay (2001) han sido criticadas por Birdsall y Hamoudi (2002) y por el (2000) cuestionan la arbitrariedad en la selección de los países, ya que ello lleva a sesgar los hallazgos a favor de los globalizantes:

- En primer lugar, Dollar y Kraay (2001) combinan una medida de política comercial, con un resultado (comercio/PBI) para seleccionar a los países. Rodrik (2000) sostiene que ello no es apropiado desde el punto de vista de los diseñadores de política, pues estos no controlan directamente el nivel de comercio, las herramientas a disposición de los gobiernos son los aranceles y los obstáculos no arancelarios, pero no los niveles de exportaciones e importaciones.

- En segundo lugar, y usando la misma data, seleccionar a los países que se ubican en el $40 \%$ superior en términos de amplias reducciones en los aranceles, así como fuentes aumentos en el ratio comercia/PBI para los periodos 1980-1984 y 19951997. Las globalizantes serían Argentina, Brasil, Colombia, Haití, Hungría, Jamaica, Corea, México, Marruecos, Malasia, Filipinas, Paraguay, Tailandia, y Uruguay. La experiencia de crecimiento de estos países presenta un resultado muy distinto del reportado por Dollar y Kraay (2001).

- En tercer lugar, toma a los diez países con mayor reducción arancelaria desde 1980: Brasil, Colombia, Haití, Uruguay, Sudáfrica, Chile, Kenia, Ghana. Este grupo de globalizante fue el que mostró el mayor colapso en el PBI a inicios de década de 1980.

- En cuarto lugar, la India y la China procedieron a realizar las reformas comerciales una década después de mostrar un crecimiento sostenido; de ahí que la pregunta relevante para los diseñadores de política no sea si el comercio en sí mismo es positivo o negativo, sino la secuencia correcta de políticas y el grado de prioridad recibido por las profundas liberalizaciones comerciales al juicio del proceso de reformas.

\section{INTEGRACIÓN Y CRECIMIENTO}

- Por el lado de la liberación financiera, debería manifestarse en un aumento de los flujos privados de capital de los países ricos hacia los pobres. Con más capital, las opciones de crecimiento son mayores, dado el trabajo nivel de ahorro interno que muestran. En otras palabras, la apertura financiera debería llevar a un mayor crecimiento, gracias al influjo de capitales. Sin embargo, la apertura financiera abrupta, en especial a los capitales de corto plazo, ha generado una serie de crisis, con efectos devastadores sobre el PBI y la pobreza. 


\section{Abel Andrés Zavala / Ciro E. Calero Castillo}

- Un problema clave asociado con la apertura financiera es que el exceso a los mercados mundiales de capitales tiende a ser asimétrico. La mayoría de los países en desarrollo accede a los mercados de capitales solo en los buenos tiempos; mientras que en los otros, enfrentan restricciones en el acceso. De este modo, el acceso es procíclico, por lo que las tendencias del crecimiento asociadas seguirán el mismo patrón.

- Desde luego, esto no implica que el ingreso de capitales sea negativo. Por el contrario. Los países pobres lo requieren en condiciones de fluidez y estabilidad. Algunos tipos de ingresos de capitales sea negativo, por lo contrario, los países pobres lo requieren en condiciones de fluidez y estabilidad. Algunos tipos de ingreso de capitales, como la inversión extranjera directa, se ajustan mejor a las dos condiciones mencionadas. Nuevamente, e igual que en el caso de la liberación comercial, sí importa la secuencia en la apertura y prioridad otorgada a la liberación rápida. La experiencia internacional de las últimas décadas es ilustrativa al respecto.

\section{CRECIMIENTO ECONÓMICO Y REDUCCIÓN DE LA POBREZA}

Desde el punto de vista histórico, el crecimiento económico no ha mostrado una tendencia lineal. En el Cuadro N. $^{\circ} 7$, se muestra las tasas de crecimiento por periodo y para diferentes regiones:

- En primer lugar, y como tendencia global, el crecimiento económico mundial ha atravesado por dos periodos de aceleración: entre 1870-1913 (1.30\% promedio anual), entre 1950-1973 (2.92\% promedio anual).

- En segundo lugar, durante las dos olas globalizadoras, las tasas de crecimiento mundial han sido relativamente similares (1.30\% entre 1870 y 1930; 41,41\% entre 1973 y 2001).

- En tercer lugar, el periodo de entre guerras muestra una notable desaceleración.

- En cuarto lugar, existen fuertes disparidades regionales. El rezago de África a lo largo de la historia es claro. A partir de 1950, así experimenta un salto hacia el crecimiento que se acentúa entre 1973 y 2001. América Latina crece por encima del promedio mundial solo en dos periodos: 1870-1913 y 1913-1950. En la misma línea destaca el lento crecimiento de Europa Oriental. América Latina y África durante la segunda globalizadora (1973-2001). 


\section{Globalización económica y pobreza}

Como análisis, en cada periodo han habido regiones que han sido capaces de crecer y prosperar más que otras.

- En los cuadros N. ${ }^{\circ} 1$ y 2 se muestran la evolución del porcentaje de pobres en el mundo. Entre 1870 y 1910, disminuyó de $89.6 \%$ a $82.4 \%$, mientras que la economía mundial crecía a un promedio anual de 1.3\%. La tendencia hacia la revolución de la pobreza continuó entre 1913 y 1950, a pesar del lento crecimiento experimentado en el periodo. Entre 1950 y 1973, etapa conocida como la "Edad de Oro del Capitalismo", la economía mundial creció a una tasa promedio anual de 2,92\% (la mayor de la historia) y la pobreza se redujo de $71.9 \%$, en 1950 , a $60.1 \%$, en 1970 ; fue la mayor reducción el porcentaje de pobres. Por último, entre 1973 y 2001, en menor crecimiento mundial (1.41\% promedio anual) En comparación con la etapa previa, se reflejó en una disminución más lenta de la pobreza.

Cuadro N. $^{\circ}$ 7. Tasas de crecimiento en el PBI por habitante (1820 - 2001) en porcentaje

\begin{tabular}{|l|r|r|r|r|}
\hline \multicolumn{1}{|c|}{ PAÍs } & $\mathbf{1 8 2 0} \mathbf{- 1 8 7 0}$ & $\mathbf{1 9 1 3} \mathbf{- 1 9 5 0}$ & $\mathbf{1 9 5 0} \mathbf{- 1 9 7 3}$ & $\mathbf{1 9 7 3} \mathbf{- 2 0 0 1}$ \\
\hline Europa Occidental & 0.95 & 0.76 & 4.05 & 1.88 \\
\hline Japón & 0.19 & 0.89 & 8.06 & 2.14 \\
\hline Europa Oriental & 0.63 & 0.60 & 3.80 & 0.68 \\
\hline Antigua URSS & 0.63 & 1.76 & 3.35 & -0.96 \\
\hline América Latina & 0.10 & 1.42 & 2.52 & 0.91 \\
\hline Asia (sin Japón) & -0.11 & 0.00 & 2.92 & 3.55 \\
\hline África & 0.12 & 1.02 & 2.07 & 0.19 \\
\hline Mundo & 0.53 & 0.91 & 2.92 & 1.41 \\
\hline
\end{tabular}

Fuente: Maddison (2003).

El crecimiento económico, ¿reduce la pobreza? De acuerdo con las tenencias mostradas, y en general, la respuesta es afirmativa. Sin embargo, la integración, como se ha mencionado, altera el estilo de crecimiento del país. De allí que genere ganadores y perdedores. 


\section{Abel Andrés Zavala / Ciro E. Calero Castillo}

El crecimiento económico es bueno e imprescindible para la reducción de la pobreza, pero no es suficiente. Sin crecimiento es imposible que los ingresos de los pobres aumenten de manera sostenida. Sin embargo, para cualquier nivel de ingresos de un país, la incidencia sobre la disminución de la pobreza está determinada por la distribución de ingresos. Dicho de otra forma, la distribución de los beneficios del crecimiento sí importa. Los países con una distribución de ingresos más desigual deben crecer a una tasa mayor que aquellos con una distribución de ingresos más equitativa, para lograr los mismos impactos en los aumentos de los ingresos de los pobres.

\section{CONCLUSIONES}

- Desde el siglo XIX se han dado dos olas globalizadoras:

La Primera se dio entre los años 1870-1913; mientras que la Segunda, desde 1980 hasta la actualidad. Durante la primera ola globalizadora, el PBI por habitante, creció a una tasa promedio anual de $1.32 \%$; y el número de pobres aumentó de 1.134 millones, en 1870 , a 1.416 millones (Cuadro N. ${ }^{\circ} 1$ ).

- Por otro lado se verifica una tendencia similar; pues el número de pobres aumentó de 2.450 millones, en 1981, a 2.735 millones, en 2001 (ver Cuadro N. ${ }^{\circ}$ 6); mientras que el PBI por habitante, aumentó a una tasa promedio anual de $1.41 \%$. También es cierto que si la medición se hace en términos porcentuales; en ambos periodos, el porcentaje de pobres disminuyó.

- Lo más resaltante de la conclusión se arriba en que:

a) El estudio de la relación entre globalización económica y pobreza no puede dejar de lado al crecimiento demográfico.

b) La vinculación entre las dos variables mencionadas está plagada de problemas empíricos. Esto es esperable, tanto por las dificultades de medición como por la posibilidad de aislar los impactos de alguna de las dimensiones de la globalización económica sobre la pobreza.

c) Si se acepta que el crecimiento económico es bueno para reducir la pobreza, surgen una serie de cuestiones. A juzgar por los resultados mostrados, el crecimiento en ambas olas globalizadoras, o no ha sido suficiente o no se ha distribuido de manera equitativa. 


\section{Globalización económica y pobreza}

Por otro lado el crecimiento asiático, durante el periodo 1973 - 2001, ¿̇estuvo basado en el libre mercado? Existe una buena cantidad de estudios que demuestran que, al menos, en un principio, no fue así. La clave estuvo en la orientación hacia fuera, es decir, un estudio de crecimiento basado en las exportaciones de bienes con valor agregado. Y ello estuvo originado en una particular combinación de políticas públicas con el uso del mercado. En otras palabras, Asía Oriental se integró al mundo con éxito, manteniendo la disciplina macroeconómica, pero con un Estado promotor, es decir, un Estado funcional al mercado.

Se puede mencionar que el meollo de la cuestión está en estudiar a la globalización económica y su relación con la pobreza desde la perspectiva del desarrollo; ya que no solo son las políticas que aceleran el crecimiento, sino también cómo el proceso político puede producir los cambios en las políticas. Y a pesar de los múltiples estudios existentes en este campo todavía hay mucho por descubrir.

\section{BIBLIOGRAFÍA}

Acosta, Alberto (2003). Globalización o desglobalización.

Adelman, Irma (1998). Evolución socioeconómica en la Europa capitalista.

Aninat, Eduardo (2002), Cómo enfrentar los desafíos de la globalización. Finanzas y desarrollo.

Banco Interamericano de Desarrollo - BID (2003).

Banco Mundial (2003). Desigualdad en América Latina y el Caribe.

Blaug, Mark (1985). Teoría Económica en retrospección. Primera edición en español, de la tercera en inglés. México: Fondo de Cultura Económica.

Callincos, Alex (2001). Contra la tercera vía. Una critica alternativa. Barcelona: Editorial Crítica.

Cárdenas, Enrique; Ocampo, José Antonio y Rosemary (2003). La era de las exportaciones latinoamericanas. De fines del siglo XIX a principios del siglo XX. México: Fondo de Cultura Económica.

CEPAL (2004). Panorama social de América Latina 2004, Santiago de Chile. 


\section{Abel Andrés Zavala / Ciro E. Calero Castillo}

CEPAL (2003). Panorama social de América Latina.

CEPAL (2002). Globalización y desarrollo. Santiago de Chile.

Chacholiades Miltiades (1981). Economía internacional. México.

Chen, Shaohur y Martín Ravallion (2004). Análisis y historia de la globalización.

Londoño, Juan Luis (2002). "Comercio, recursos y desigualdad en América Latina. Revista de la CEPAL N. ${ }^{\circ}$ 80. Santiago de Chile.

Niveau, Maurice (1985). Historia de los hechos económicos contemporáneos, 9. a ed. en español. Barcelona: Ariel Economía. 